\title{
The Effects of High-Frequency Residual Currents on the Operation of Residual Current Devices
}

\author{
T. M. H. Slangen, B. R. F. Lustenhouwer, V. Ćuk and J. F. G. Cobben \\ Department of Electrical Energy Systems \\ Eindhoven University of Technology \\ $5600 \mathrm{MB}$, Eindhoven, The Netherlands \\ E-mail: t.m.h.slangen(at)tue.nl
}

\begin{abstract}
This research investigates the effects of high frequency currents between $50 \mathrm{~Hz}$ and $150 \mathrm{kHz}$ on the operation of Residual Current Devices (RCDs). Nowadays, the increasing amount of large power-electronic switching devices can be a source of both harmonics $(<2 \mathrm{kHz})$ and supraharmonics $(2-150$ $\mathrm{kHz}$ ) currents injected to the grid. This can have several effects and possibly lead to unwanted tripping of RCDs, due to high earth-currents that can be emitted by the devices. The question is if supraharmonics can also lead to misoperation or fail-to-operate conditions for the RCDs, potentially leading to serious safety risks. A set-up is developed to introduce both $50 \mathrm{~Hz}$ and highfrequency leakage currents. First, the $50 \mathrm{~Hz}$ tripping-current of the RCDs is tested under nominal conditions. Secondly, the tripping current for non-nominal frequencies (between $50 \mathrm{~Hz}$ and $150 \mathrm{kHz}$ ) is determined to verify the possibility for false tripping. Lastly, the $50 \mathrm{~Hz}$ tripping current for the RCD is tested in the presence of a high-frequency current. The most important conclusion is that RCDs of type A and AC have an increased fundamental $(50 \mathrm{~Hz})$ tripping current when there are HFcomponents present. This potentially results in a safety risk.
\end{abstract}

Key words. Residual current device, power system harmonics, supraharmonics, electrical safety, earth fault current.

\section{Introduction}

The increasing number of devices with high-power rectifiers and inverters like electric vehicle chargers and photovoltaic (PV) inverters, also increases the amount of harmonic and supraharmonic currents in the low-voltage grids. Supraharmonic (SH) distortion is the name of all conducted waveform distortion within the frequency range of $2 \mathrm{kHz}$ to $150 \mathrm{kHz}$ [1], [2].

Previous research introduced the possibility that highfrequency (HF) leakage currents above $50 \mathrm{~Hz}$ can lead to the unwanted tripping (false positive) of residual current devices (RCDs) [3],[4]. In the case when multiple supraharmonic frequency components at almost the same frequency are present, this results in a so-called frequency beating effect that creates additional low-frequency components $(\mathrm{Hz}$ to tens of $\mathrm{Hz}$ ) that may be the source of the unwanted tripping as well [5].

Another possibility is that the RCDs may be vulnerable to high frequency components, as they could potentially lead to situation where the RCD does not trip in the presence of an earth fault current [6]. Where the unwanted tripping (false positive) is annoying and can lead to unwanted interruptions of the power supply, a situation where an RCD does not trip (false negative) can lead to dangerous situations in which the protection for humans and animals against harmful currents then fails.

To goal of this research is to investigate the possibilities for both the false positive as the false negative situations for RCDs. The effects of high-frequency (HF) currents above the fundamental current of the RCD $(50 \mathrm{~Hz})$ on the tripping behaviour is analysed. To achieve this, a set-up is developed to introduce fundamental $(50 \mathrm{~Hz})$ and highfrequency $(>50 \mathrm{~Hz})$ leakage currents to an RCD simultaneously. The test set-up consists of two function generators, two amplifiers and 2 loads. Additionally, a nominal $230 \mathrm{~V}, 50 \mathrm{~Hz}$ circuit consisting of a household load is connected to create a representative situation and to make sure that voltage-dependent RCDs can also trip. The tests are carried out on 5 RCDs of different types and from different manufacturers.

This paper is structured as follows; first, in section 2 background on RCDs is given and the differences between the types are explained. Next, in section 3 the methodology and test procedure are presented, where section 4 discusses the most important results. Finally, section 5 presents the conclusion and in section 6 recommendations for further research are given.

\section{Theoretical background}

The purpose of an RCD is to detect earth fault currents and to prevent humans (and animals) from dangerous currents through the body. A continuous current of more than $30 \mathrm{~mA}$ at $50 \mathrm{~Hz}$ flowing through the human body for more than $300 \mathrm{~ms}$ can cause irreversible damage to the normal cardiac cycle (ventricular fibrillation) and higher currents or exposure times even death (electrocution) [7]. For this reason, RCDs that serve protection of humans should always operate within $300 \mathrm{~ms}$ and at a maximum current of $30 \mathrm{~mA}$. An RCD measures the sum of all currents flowing into an installation and the returning neutral current. This sum is in a normal situation without earth leakage equal to $0 \mathrm{~mA}$. According to IEC 61008 [8] an RCD should break the circuit between $0.5 I_{\Delta n}$ and $I_{\Delta n}$, with $I_{\Delta n}$ being the rated tripping current of the RCD. For human protection to earth fault currents a rating of maximum 30 
$\mathrm{mA}$ is required, meaning the RCD should trip between 15 and $30 \mathrm{~mA}$, after a maximum time of $300 \mathrm{~ms}$.

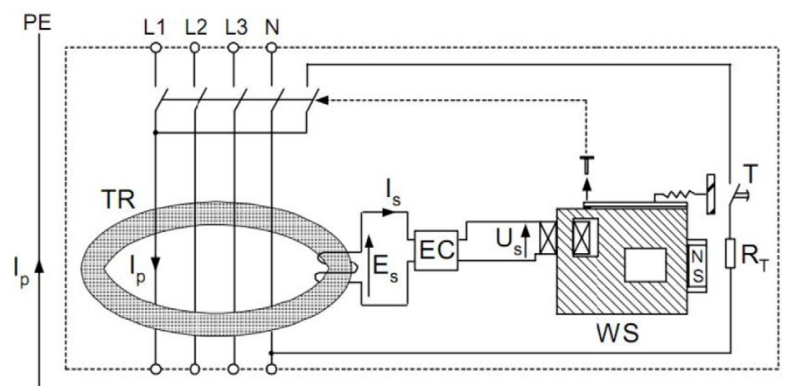

Fig. 1. A simple overview of a 4-pole RCD, showing the differential transformer (TR), permanent magnet (NS) and electromagnetic relay (WS) [3].

In some installations RCDs with a $300 \mathrm{~mA}$ rating are used for protection against fires, but they are inadequate in providing personal protection in the case of a direct contact. An RCD may also provide an integral overcurrent protection circuit, combining the function a miniature circuit breaker (MCB) and an RCD into one device. This is then called an RCBO (Residual Current Breaker + Overcurrent protection). The RCDs tested in this research did not have an integral overcurrent protection circuit and are sometimes also called an RCCB (Residual Current Circuit Breaker). In the rest of this paper simply RCD will be used.

For higher frequencies than $50 \mathrm{~Hz}$, it is assumed that the probability of ventricular fibrillation decreases with increasing frequency, as in IEC standard 60479 [9]. Here a so-called frequency factor $F_{f}$ is proposed with which the maximum allowable tripping current of $30 \mathrm{~mA}$ should be multiplied. For example, for a frequency of $200 \mathrm{~Hz}$ this factor is 2.0, meaning a maximum current of $F_{f} I_{\Delta n}=60 \mathrm{~mA}$ is allowed. This factor is defined for frequencies up to 1 $\mathrm{kHz}$, and some values are shown in Table I. This factor is calculated by assessing the impact of currents on the human body for different frequencies [4]. When assessing if an RCD compromises a possibly safety issue for higher frequencies than $50 \mathrm{~Hz}$, this should be considered.

Table I. - Frequency factor $F_{f}$ as defined in IEC 60479 and

\begin{tabular}{l|l|l|l|l|l|l}
\multicolumn{8}{c}{ calculated $F_{f} I_{\Delta n}$} \\
$f(\mathrm{~Hz})$ & 100 & 200 & 300 & 400 & 500 & 600 \\
\hline$F_{f}(-)$ & 1.5 & 2.0 & 4.5 & 5.8 & 7.0 & 9.2 \\
\hline$F_{f} I_{\Delta n}(\mathrm{~mA})$ & 45 & 60 & 135 & 174 & 210 & 276
\end{tabular}

A simplified overview of an RCD is shown in Fig. 1. An RCD normally contains a differential transformer (TR) with a high permeability magnetic core and one primary winding around all phases and neutral, a permanent magnet (N-S) and an electromagnetic relay (WS). The permanent magnet will create a constant magnetic field, counterbalancing the force of the spring holding the switch in place. When a residual current $I_{\Delta n}$ is present, a magnetic flux will be induced by the differential transformer, resulting in a reduced nett magnetic field and finally tripping of the RCD [3]. There are different types of RCDs of which the three most-used types for domestic installations are studied in this research. These types are [10];
- Type AC: Detects alternating sinusoidal currents, suddenly applied or smoothly increasing. Presently not advised (even not allowed in NL) as it is unable to detect pulsed fault currents.

- $\quad$ Type A: Same as type AC and in addition also detects residual pulsed direct current (PDC). Mostly applied in residential installations.

- Type B: Same as type A and in addition also detects non-pulsating (smooth) direct currents (DC). Recommended in installations with PV-inverters or $\mathrm{EV}$-chargers where no further appropriate protection against DC fault currents ( $>6 \mathrm{~mA})$ is provided.

The specifications of the RCDs used in this research, of 4 different renowned manufacturers, are shown in Table II. The value $I_{n}$ represents the rated current of the RCD and $I_{\Delta n}$ the rated residual current or tripping current. All RCDs have 4-poles, to be used in a three-phase electric circuit including neutral.

Table II. - The RCDs used in this research. $I_{n}$ represents the rated current and $I_{\Delta n}$ the rated residual current or tripping

\begin{tabular}{l|lllll} 
& \multicolumn{5}{c}{ current. } \\
RCD \# & 1 & 2 & 3 & 4 & 5 \\
\hline$I_{n}(\mathrm{~A})$ & 40 & 40 & 40 & 25 & 25 \\
$I_{\Delta n}(\mathrm{~mA})$ & 300 & 300 & 30 & 30 & 30 \\
Type & B & AC & A & B & AC
\end{tabular}

\section{Methodology}

In line with the research goals, a test set-up enabling the simultaneous injection of $50 \mathrm{~Hz}$ fundamental leakage currents $\left(I_{\Delta \mathrm{n}}\right)$ and a high-frequency $(\mathrm{HF})$ leakage current $\left(I_{\Delta \mathrm{HF}}\right)$ is developed. For simplicity, only 4-pole RCDs are used. The RCDs have separate connections for every phase $(\mathrm{L} 1, \mathrm{~L} 2, \mathrm{~L} 3)$ and neutral $(\mathrm{N})$, as shown in Fig. 1. Because they have a combined measurement of the phases using the primary winding, the leakage currents can be injected on one of the phases, not returning via the neutral. In practice, the high-frequency leakage current will be superimposed on the fundamental current, but the assumption is that this does not affect the RCD differently. To ensure the RCD is always energized, the nominal $50 \mathrm{~Hz}, 230 \mathrm{~V}$ circuit fed from the mains is connected between phase 1 and the neutral, feeding a primarily resistive load of about $50 \mathrm{~W}$.

\section{A. Test set-up}

The test set-up consists of 2 function generators to create the fundamental- and the HF leakage current, respectively. To amplify the signals, 2 power amplifiers are used. For the $50 \mathrm{~Hz}$ leakage currents the custom built THE EM-1698 (by TU/e, formerly called THE) with a $20 \mathrm{kHz}$ bandwidth and adjustable gain is used. For the HF leakage current an amplifier with an $1 \mathrm{MHz}$ bandwidth and a constant gain of 10 is used. Both are connected to a resistive load and the current amplitudes are varied by controlling the function generators. A schematic of the setup is shown in Fig. 2, here the function generators and amplifiers are depicted as current sources. The RCD is shown as a 4-pole switch. The currents are measured with high-accuracy current transducers and an oscilloscope. To ensure the energized conditions needed for some RCDs to trip, a separate circuit between phase 1 and neutral is 
always connected, resulting in a non-residual current of $220 \mathrm{~mA}$. Here no differential current is created.

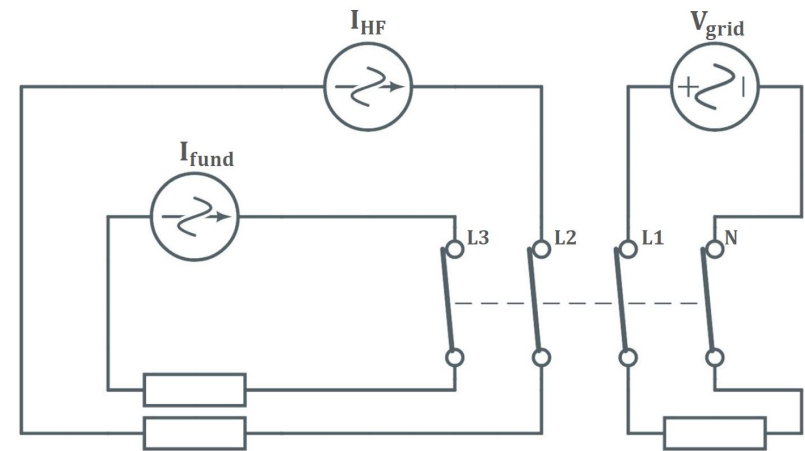

Fig. 2. Schematic of the test set-up. $I_{\text {fund }}$ is the created fundamental $(50 \mathrm{~Hz})$ leakage current, $I_{H F}$ the high-frequency leakage current, both non-returning via the RCD. The mains $V_{\text {grid }}$ energizes the RCD and feeds a small resistive load.

\section{B. Experiments}

On every RCD different experiments are performed and repeated 10 times to determine the standard deviation of the measurement outcomes. Experiment A has the goal to verify the nominal $I_{\Delta n}$ value of the RCD to see if the RCD can operate under normal conditions according to the specifications. Then, in experiment B, a HF leakage current of different frequencies and amplitudes is created to see the behaviour for HF and the possibility for a false positive. The maximum frequency for which the RCD is sensitive is determined. Experiment $\mathrm{C}$ is used to see if a combination of a fundamental and a HF leakage current can result in a false positive (or false tripping) due to an increased composite current $\left(I_{\text {fund }}+I_{H F}\right)$. Experiment D is similar to experiment $\mathrm{C}$, but here the possibility for a false negative (also called blinding) in which the fundamental tripping current is increased is tested for frequencies outside the tripping range as determined in experiment B. In this research, the time-to-trip is not considered. For this reason, every current level step was maintained for at least 2 seconds (above the instantaneous tripping time of $300 \mathrm{~ms}$ for the RCDs). The different experiments will be described in more detail.

1) Experiment $A$ : The RCD is energized with both sources turned off. Then, in steps of $1 \mathrm{~mA}$ a fundamental leakage current is created $\left(I_{\text {fund }}\right)$, starting at $0.4 \cdot I_{\Delta n}$ until the RCD trips. The value is registered as $I_{\text {fund,trip }}$ and the test repeated 10 times.

2) Experiment $B$ : The RCD is energized with both sources turned off. Then, a HF leakage current $I_{H F}$ at a fixed frequency (starting at $100 \mathrm{~Hz}$ ) and a fixed amplitude is created. Then the amplitude of the current is increased in steps of $1 \mathrm{~mA}$ until the RCD trips. This value is registered or DNT (did not trip) in the case when the RCD did not trip for the maximum possible current, limited by the equipment. Then the source is turned off, the RCD energized again, and the experiment repeated for the next fixed frequency (increasing in steps of 50 $\mathrm{Hz}$ ) to determine the frequency range for which the RCD seems sensitive and $f_{\text {max }}$ for which the RCD never trips beyond this frequency.
3) Experiment $C$ : The RCD is energized with both sources turned off. Then, a fundamental $50 \mathrm{~Hz}$ leakage current $I_{\text {fund }}$ is created at a constant value of $0.5 I_{\Delta n}$. Then, like experiment B, a HF leakage current $I_{H F}$ with a fixed frequency and amplitude inside the range determined in experiment $\mathrm{B}$ $\left(<f_{\text {max }}\right)$ is added and increased until the RCD trips. This value is then registered, and the test repeated 10 times. The experiment proceeds similar as experiment B until $f_{\max }$ is reached.

4) Experiment $D$ : The RCD is energized with both sources turned off. Then a constant HF leakage current $I_{H F}$ of $800 \mathrm{~mA}$ is created at a fixed frequency and amplitude starting with the frequencies outside the range as determined in experiment $\mathrm{B}\left(>f_{\max }\right)$; the frequencies that resulted in a DNT for the RCD. Then, a fundamental leakage current $I_{\Delta n}$ is created and increased until the RCD trips. This value is registered, and the test repeated 10 times. Then the frequency is increased in steps up to $150 \mathrm{kHz}$ and for each frequency the test is repeated 10 times. Finally, the amplitude of $I_{H F}$ is increased to 1300 $\mathrm{mA}$ and the experiment repeated.

Each measurement is conducted at least 10 times for every RCD to determine the mean and standard deviation of the measurement outcomes. This is afterwards used to determine if any observed deviating values between experiments should be deemed significant or not.

\section{Results}

In this section the most important results will be discussed for every experiment A-D.

\section{A. Determination of $I_{\text {fund,trip }}$}

In Fig. 3. the results of experiment A are shown. According to the standard all RCDs should trip between $0.5 I_{\Delta n}$ and $I_{\Delta n}$, where the rated residual current equals 300 $\mathrm{mA}$ for RCD 1 and 2, and $30 \mathrm{~mA}$ for RCD 3, 4 and 5. All tested RCDs were able to trip within the specifications as seen in the figure. The boxplot shows the median value, the spread of the measurement outcomes and the outliers.
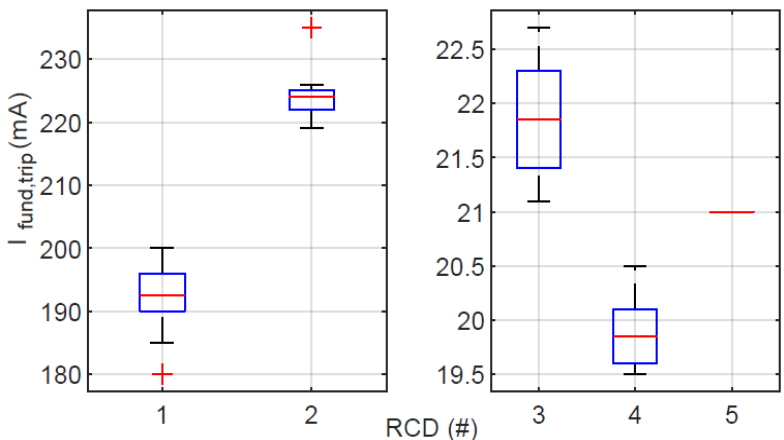

Fig. 3. Results of Experiment A, determination of nominal tripping behaviour for a fundamental $50 \mathrm{~Hz}$ leakage current $\left(I_{\text {fund,trip }}\right)$. All RCDs trip within their specification $(300 \mathrm{~mA}$ for RCD 1-2, $30 \mathrm{~mA}$ for RCD 3-5) with some variance. 


\section{B. HF trip sensitivity and determination of $f_{\max }$}

In this experiment the HF trip sensitivity of the RCDs was tested. In Fig. 4 the median value of the current that was needed to trip the $\operatorname{RCD}\left(I_{H F, \text { trip }}\right)$ is shown for different frequencies. It is observed that for increasing frequencies, the leakage current needed to trip the RCD is increasing. For the RCD-3 (A-30) a current of $2100 \mathrm{~mA}$ was needed before tripping occurred at $500 \mathrm{~Hz}$ (not in figure).

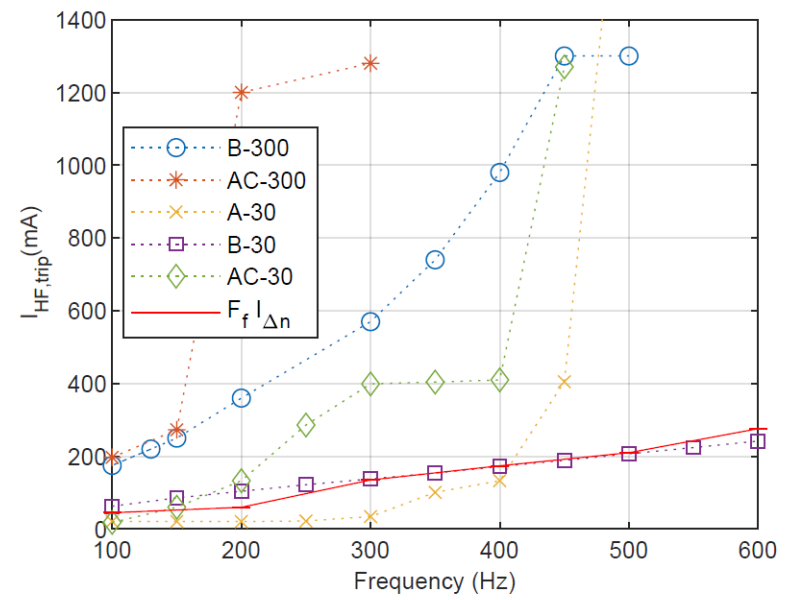

Fig. 4. Median value of measured HF tripping current of the RCDs when exposed to a leakage current with a frequency between 100 and $600 \mathrm{~Hz}$ and $F_{f} I_{\Delta n}$ according to Table I.

Table III. - Maximum frequencies for which the RCDs tripped

\begin{tabular}{l|l|l|l|l|l} 
RCD \# & 1 & 2 & 3 & 4 & 5 \\
\hline$f_{\max }(\mathrm{Hz})$ & 500 & 300 & 500 & N/D & 450
\end{tabular}

The RCDs in this figure show different $f_{\text {max }}$ values as shown in Table III, indicating they do not trip for frequencies above this value. An exception is RCD-4 which tripped for all frequencies up to $150 \mathrm{kHz}$, and hence the maximum frequency for this RCD could not be determined (N/D). For this RCD, the measured tripping current for different frequencies between $100 \mathrm{~Hz}$ and 150 $\mathrm{kHz}$ is shown in Fig. 5. The current needed to trip this RCD increases up to $1660 \mathrm{~mA}$ at $8 \mathrm{kHz}$, then decreases to a value of $654 \mathrm{~mA}$ at $18 \mathrm{kHz}$ and continues to increase almost linearly up to $130 \mathrm{kHz}$. Then the curve flattens, and the RCD needs $2540 \mathrm{~mA}$ to achieve tripping at a frequency of $150 \mathrm{kHz}$. This behaviour might be further explained by looking into the components of a type-B RCD as it contains an extra sensing circuit compared to the types $\mathrm{A}$ and $\mathrm{AC}$ [11]. This, however, is out of scope for this research.

When comparing the values found in Fig. 4 with the calculated values for $F_{f} I_{\Delta n}$ in Table I, it is concluded that for the higher frequencies RCD A-30 can trip for values that are considered safe, but only up to $400 \mathrm{~Hz}(<174 \mathrm{~mA})$. RCD B-30 only responds correctly for frequencies above $400 \mathrm{~Hz}$ but at too high values for frequencies between 100 and $400 \mathrm{~Hz}$. The RCD AC-30 trip at higher values for all frequencies which can result in a safety issue in the case when a leakage current with a frequency above $50 \mathrm{~Hz}$ is present. The RCDs of the $300 \mathrm{~mA}$ type are not considered as they are not meant to be used for human protection against direct contact.

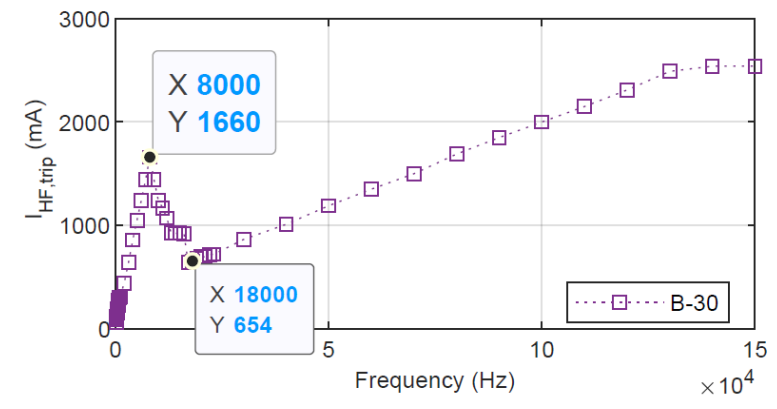

Fig. 5. Median value of measured tripping current of RCD-4, when exposed to a leakage current with a frequency between $100 \mathrm{~Hz}$ and $150 \mathrm{kHz}$.

C. HF sensitivity with $f_{H F}<f_{\text {max }}$ in the presence of $0.5 I_{\Delta n}$ When combining both the leakage currents from $I_{\text {fund }}$ and $I_{H F}$ for frequencies between $50 \mathrm{~Hz}$ and $f_{\text {max }}$ different results are obtained compared to experiment B. For the RCDs with a rated residual current of $30 \mathrm{~mA} \mathrm{(3-5)} \mathrm{a}$ constant fundamental residual current $I_{\text {fund }}$ of $15 \mathrm{~mA}$ was created, and $150 \mathrm{~mA}$ for the $300 \mathrm{~mA}(1-2)$ variants. The tripping currents in this case for the different frequencies are shown in Fig. 6. The figure shows the measured HF current $I_{H F}$ at which the RCD tripped $\left(I_{H F, \text { trip }}\right)$. RCDs B300, AC-300 and B-30 tripped for lower values over the whole frequency range, and A-30 and AC-30 only for part of the frequencies. There is however no clear relation between the decrease in tripping current and the total leakage current that was measured by the RCD; $\left(I_{\text {fund }}+\right.$ $\left.I_{H F}\right)$.

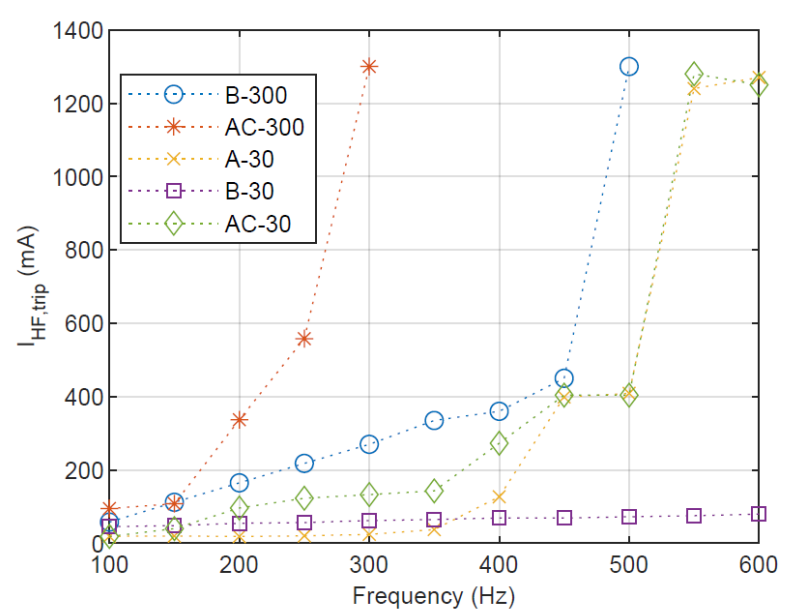

Fig. 6 Median value of measured HF tripping current of the RCDs when exposed to both a HF leakage current between 100 and $600 \mathrm{~Hz}$ and a fundamental leakage current of $0.5 I_{\Delta n}$ at 50 Hz. Note: the HF tripping current is shown, not the composite. 

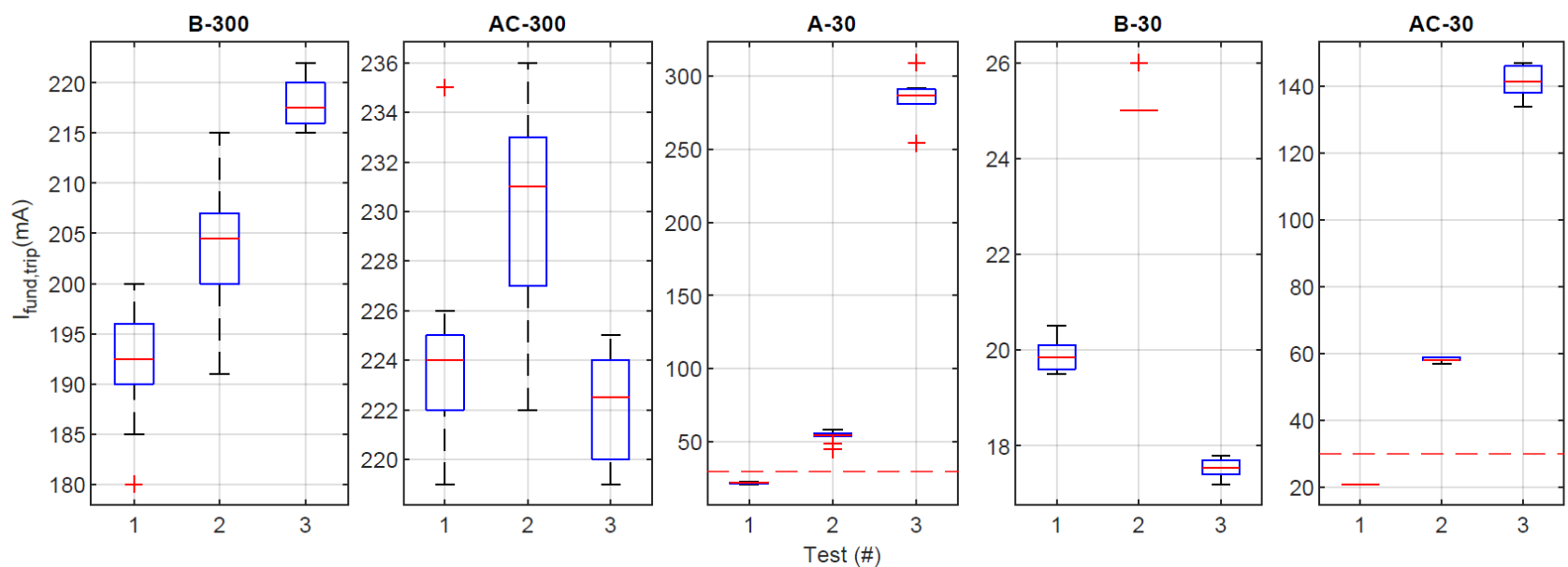

Fig. 7. Measured fundamental $(50 \mathrm{~Hz})$ tripping current $\left(I_{\text {fund,trip }}\right)$ of the $5 \mathrm{RCDs}$ in a normal situation (Test 1 , result from Experiment A), in the presence of an $800 \mathrm{~mA}, 1 \mathrm{kHz}$ leakage current (Test 2) and in the presence of a $1300 \mathrm{~mA}, 1 \mathrm{kHz}$ leakage current (Test 3). All measurements are repeated 10 times. The RCDs indicated as A-30 and AC-30 fail to trip within their rated maximum tripping current of 30 $\mathrm{mA}$. The other RCDs are also affected but still within specification and less significant.

\section{Determination of $I_{\text {fund,trip }}$ in the presence of $I_{H F}$ with $f>f_{\max }$}

In this experiment HF leakage currents with frequencies above the determined $f_{\max }$ (except for RCD4) are created. The RCDs that where affected the most where the types A-30 and AC-30 and the results are shown in Fig. 8. Here the calculated median values are used following the 10 outcomes for each test. In this figure, the red line indicates the value at which the RCDs should ultimately trip; $30 \mathrm{~mA}$ in both cases. In Fig. 8 (a) the measured fundamental tripping current $I_{\text {fund,trip }}$ is shown when a HF leakage current of $800 \mathrm{~mA}$ was injected. An increase in the tripping current beyond the maximum value is observed for frequencies up to $10 \mathrm{kHz}$. The maximum tripping value for $\mathrm{A}-30$ is raised to $76 \mathrm{~mA}$ at $5 \mathrm{kHz}$ and 58 $\mathrm{mA}$ at $1 \mathrm{kHz}$ for $\mathrm{AC}-30$.
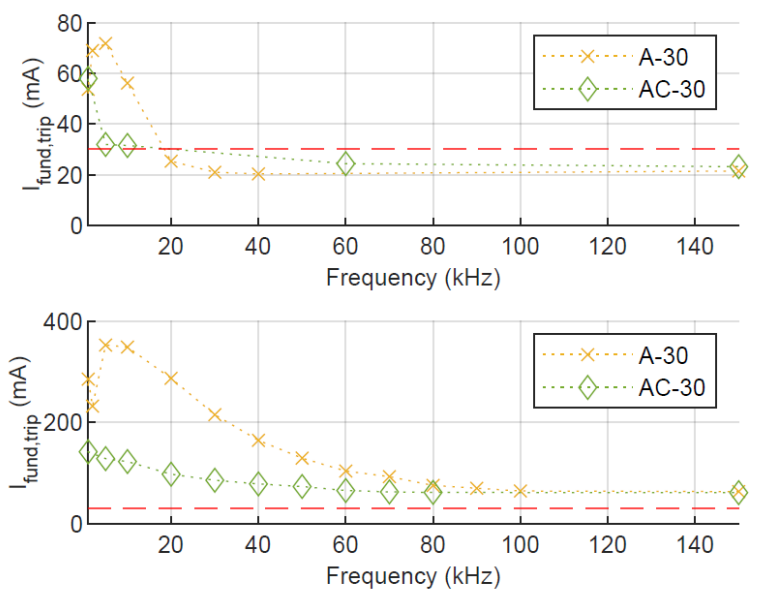

Fig. 8. Median value of measured fundamental $(50 \mathrm{~Hz})$ tripping current $\left(I_{\Delta n}\right)$ of RCD-3 and RCD-5 when exposed to an $800 \mathrm{~mA}$ (a) or $1300 \mathrm{~mA}$ (b) leakage current at variable frequency between $1 \mathrm{kHz}$ and $150 \mathrm{kHz}$.

When increasing the HF leakage current in amplitude up to $1300 \mathrm{~mA}$, the results are shown in Fig. 8 (b). The increase in nominal tripping current is now even higher, up to 350 $\mathrm{mA}$ for A-30 and $141 \mathrm{~mA}$ for AC-30. This potentially results in a safety issue as these RCDs allow $50 \mathrm{~Hz}$ leakage currents with dangerously high amplitude to flow for seemingly indefinite time.

To see if this increase is also significant for the other RCDs, a statistical analysis of the measurements is done. The results are shown in Fig. 7. Here for each RCD 3 different boxplots are shown. The first (Test 1) shows again the outcome of experiment 1 in which the nominal tripping current under normal circumstances was determined. The second and third shows the determined value of $I_{\text {fund,trip }}$ in the presence of a fixed HF component at $1 \mathrm{kHz}$ at either $800 \mathrm{~mA}$ (Test 2) or $1300 \mathrm{~mA}$ (Test 3).

The first RCD of type B-300 shows an increase in tripping current, but still within the rated value of $300 \mathrm{~mA}$. Considering the variance of the measurement values and the probability that the increase has to do with external effects, it is concluded that the increase is significant for Test 3 but not for Test 2 as there is still an overlap in measurement values with Test 1 , making the difference rather small. The RCDs of type AC-300 and B-30 are affected slightly, but with questionable significance as here the ranges also overlap with Test 1 and the differences are very small (median difference below $7 \mathrm{~mA}$ ).

The RCD of type A-30 however shows a serious increase in tripping current, as shown previously in Fig. 8. All measurement values for Test 2 and Test 3 are above the rated $30 \mathrm{~mA}$. This implies that this RCD allows fundamental $50 \mathrm{~Hz}$ leakage currents of $60 \mathrm{~mA}$ (Test 2) and $280 \mathrm{~mA}$ (Test 3) to flow for indefinite time. As seen in [9] and in Section 2, currents of $50 \mathrm{~mA}$ at $50 \mathrm{~Hz}$ can cause irreversible damage to the cardiac cycle $(>200 \mathrm{~ms})$ or result in electrocution $(>1 \mathrm{~s})$. For currents of $200 \mathrm{~mA}$ at $50 \mathrm{~Hz}$ shorter exposure times already result in irreversible damage ( $>40 \mathrm{~ms}$ ) or electrocution $(>300 \mathrm{~ms})$.

The RCD of type AC-30 also slows an increase in the tripping current. For both Test 2 and Test 3 the maximum value of $30 \mathrm{~mA}$ is exceeded, and it allows $50 \mathrm{~Hz}$ residual currents of $58 \mathrm{~mA}$ and $142 \mathrm{~mA}$ respectively for indefinite time. Again, this compromises a serious safety issue for this RCD, as it is unable to operate in the case of a dangerous leakage current present. 


\section{Conclusion}

The goal of this research was to investigate the effects of high-frequency (HF) residual currents between $50 \mathrm{~Hz}$ and $150 \mathrm{kHz}$ on the operation of residual current devices. The most important findings are as follows.

\section{A. Increase in fundamental tripping current due to $H F$}

The RCDs of type A-30 and AC-30 show a significant increase in fundamental tripping current in the presence of a high-frequency leakage current. This is considered as a false-negative (blinding) situation and results in a serious safety issue as $50 \mathrm{~Hz}$ currents above the safe maximum of $30 \mathrm{~mA}$ are possible. These RCDs are unable to operate properly to a fundamental $50 \mathrm{~Hz}$ leakage current of amplitudes up to even $300 \mathrm{~mA}$. It is important to mention that even though these RCDs did not trip for frequencies above $500 \mathrm{~Hz}$ - and therefore seem insensitive to them - there operation is affected by higher frequencies.

\section{B. Tripping sensitivity of RCDs to $H F$}

All tested RCDs are sensitive to part of the frequencies above $50 \mathrm{~Hz}$ and this can lead to false-positive operation. However, none of the $30 \mathrm{~mA}$ types operates according to IEC 60479, in which maximum - for humans considered safe - residual currents for frequencies between $50 \mathrm{~Hz}$ and $1 \mathrm{kHz}$ are defined. In general, a higher residual current is needed to operate the RCDs for higher frequencies. An exception is RCD B-30, that shows a decreasing tripping current for a part of the frequency range. When a combination of a fundamental leakage current and a high-frequency leakage current is injected, slightly different results are found. However, there is no clear relation between the change in tripping current and the total composite leakage current.

\section{Definition of $I_{\Delta n}$}

The relevant standards related to the assessment and detection of leakage currents need to clarify the definition of the nominal tripping-current $\left(I_{\Delta n}\right)$. It is unclear if only fundamental- or composite leakage currents (fundamental $+\mathrm{HF})$ are meant by this definition.

\section{Further research}

To achieve a more universal conclusion regarding the possible safety issues and effects for specific types of RCDs, more research is recommended. For instance, the following matters can be addressed.

- The effect of HF currents on the time-to-trip: This research did not take the time-to-trip into account. It is of interest to see if HF currents also influence the time-to-trip of the RCDs. This means that when the tripping current might be unaffected, the increase in exposure time can still lead to a potentially dangerous situation.

- Insight in- and modelling of the components of the RCD that cause the observed behaviour: This can give useful design recommendations to avoid the dangerous increase in fundamental tripping currents, especially for the type A and type AC devices.
- More insight in actual high-frequency residual currents, especially as caused by electric vehicle charging or PV installations.

- The immunity of type B RCDs: In this research the type B RCD shows limited effect. However, more experiments should be carried out on this type to conclude if the application of a type B is sufficient to avoid influences from HF components.

\section{References}

[1] J. Meyer et al., 'Overview and Classification of Interferences in the Frequency Range $2-150 \mathrm{kHz}$ (Supraharmonics)', in 2018 International Symposium on Power Electronics, Electrical Drives, Automation and Motion (SPEEDAM), Amalfi, Italy, Jun. 2018, pp. 165-170, doi: 10.1109/SPEEDAM.2018.8445344.

[2] S. K. Ronnberg, A. Gil-De-Castro, and R. MedinaGracia, 'Supraharmonics in European and North American LowVoltage Networks', in 2018 IEEE International Conference on Environment and Electrical Engineering and 2018 IEEE Industrial and Commercial Power Systems Europe (EEEIC / I\&CPS Europe), Palermo, Jun. 2018, pp. 1-6, doi: 10.1109/EEEIC.2018.8493930.

[3] Y. Xiang, V. Cuk, and J. F. G. Cobben, 'Impact of residual harmonic current on operation of residual current devices', in 2011 10th International Conference on Environment and Electrical Engineering, Rome, Italy, May 2011, pp. 1-4, doi: 10.1109/EEEIC.2011.5874683.

[4] F. Freschi, 'High-Frequency Behavior of Residual Current Devices', IEEE Trans. Power Deliv., vol. 27, no. 3, pp. 1629-1635, Jul. 2012, doi: 10.1109/TPWRD.2012.2191423.

[5] T. Slangen, T. van Wijk, V. Ćuk, and S. Cobben, 'The Propagation and Interaction of Supraharmonics from Electric Vehicle Chargers in a Low-Voltage Grid', Energies, vol. 13, no. 15, p. 3865 , Jul. 2020, doi: 10.3390/en13153865.

[6] C. Roldán-Porta, G. Escrivá-Escrivá, F.-J. CárcelCarrasco, and C. Roldán-Blay, 'Nuisance tripping of residual current circuit breakers: A practical case', Electr. Power Syst. Res., vol. 106, pp. 180-187, Jan. 2014, doi: 10.1016/j.epsr.2013.07.020.

[7] Beama, 'THE RCD HANDBOOK - GUIDE TO THE SELECTION AND APPLICATION OF RESIDUAL CURRENT DEVICES'. 2019.

[8] IEC, 'IEC 61008 - Residual current operated circuitbreakers without integral overcurrent protection for household and similar use (RCCBs).', 2010.

[9] IEC, 'IEC 60479 - Effect of current on human being and livestock', 2017.

[10] S. Czapp, 'The effect of earth fault current harmonics on tripping of residual current devices', in 2008 International School on Nonsinusoidal Currents and Compensation, Lagow, Poland, Jun. 2008, pp. 1-6, doi: 10.1109/ISNCC.2008.4627489. [11] R. Panda and J. Veeramalla, 'Behavior modeling of a type B RCD', in 1st IEEE International Conference on Power Electronics. Intelligent Control and Energy Systems (ICPEICES2016), 2016, p. 6. 